\title{
Feasibility of certified quality management in a comprehensive stroke care network using telemedicine: STENO project
}

\author{
René Handschu1,2, Mateusz Scibor ${ }^{1}$, Angela Wacker ${ }^{1}$, David R. Stark', Martin Köhrmann', \\ Frank Erbguth ${ }^{3}$, Patrick Oschmann ${ }^{4}$, Stefan Schwab', and Lars Marquardt'*
}

\begin{abstract}
Background Stroke care networks with and without telemedicine have been established in several countries over the last decade to provide specialized stroke expertise to patients in rural areas. Acute consultation is a first step in the management of stroke, but not the only one. Methods of standardization of care and treatment are much needed. So far, quality management systems have only been used for single stroke units. To the best of our knowledge, we are the first stroke network worldwide to aim for certification of a network-wide quality management system.

Methods The Stroke Network Using Telemedicine in Northern Bavaria (STENO), currently with 20 associated medical institutions, is one of the world's largest stroke networks, caring for over 5000 stroke patients each year. In 2010, we initiated the implementation of a network-wide 'total' quality management system according to ISO standard 9001:2008 in cooperation with the German Stroke Society and a third-party certification organization (LGA InterCert).

Results Certification according to ISO 9001:2008 was awarded in March 2011 and maintained over a complete certification cycle of 3 years without major deviation from the norm in three external third-party audits. Thrombolysis rate significantly increased from $8 \cdot 2 \%$ (2009) to $12.8 \%$ (2012).

Conclusions Certified quality management within a large stroke network using telemedicine is possible and might improve stroke care procedures and thrombolysis rates. Outcome studies comparing conventional stroke care and telestroke care are inevitable.

Key words: certification, quality management, stroke, stroke unit, telemedicine
\end{abstract}

\section{Introduction}

Stroke care has been changed substantially over the last twodecades due to the use of intravenous thrombolysis and stroke

Correspondence: Lars Marquardt*, Department of Neurology,

University of Erlangen-Nuremberg, Schwabachanlage 6, 91054 Erlangen, Germany.

E-mail: lars.marquardt@uk-erlangen.de

${ }^{1}$ Department of Neurology, University of Erlangen-Nuremberg, Erlangen, Germany

${ }^{2}$ Department of Neurology, Klinikum Neumarkt, Neumarkt, Germany

${ }^{3}$ Department of Neurology, Nuremberg Municipal Academic Hospital, Nuremberg, Germany

${ }^{4}$ Department of Neurology, Klinikum Hohe Warte Bayreuth, Bayreuth, Germany

Received: 30 October 2013; Accepted: 20 May 2014; Published online 4 August 2014

Conflict of interest: None declared.

Funding: Some of the costs for third body certification have been funded by a designated grant by the Ministry of Environment and Health of the State of Bavaria.

DOI: $10.1111 /$ ijs.12342 unit care as the first-line therapy for ischemic stroke with proven effect (1). Because of lack of stroke expertise in rural or regional hospitals, telemedicine was introduced to bring such expertise to patients in time. This method was found to be feasible and reliable when testing and scoring stroke patients by established stroke scales (2-4) and has been tested in pilot studies $(5,6)$. The benefit of telemedical stroke care can only be shown in the setting of a stroke care network linking hospitals together $(7,8)$. One of the key factors in these networks is standardization and quality management of the entire acute stroke care process (8).

As early as in 1966, Donabedian defined the dimensions of quality in medical care by structure, process, and outcome $(9,10)$. The concept of 'stroke units' delineates a combination of structure and care processes that helps to improve quality in stroke care by reduction of mortality and functional deficit. Subsequently, the German Stroke Society (GSS) developed a certification standard for stroke units reflecting process and structure quality of acute institutionalized stroke care (11). For quality of outcome, key indicators were defined out of a nationwide hospital-based data collection (12). Similarly, the American Stroke Association and the Joint Commission on Accreditation of Healthcare Organizations (JCAHO) have defined standards and a certification process for primary and comprehensive stroke centers including key quality measures (13-16).

Scientific work reflecting systematic quality management in acute stroke care is scarce $(13,16)$, and to the best of our knowledge there is no publication on its use in telemedical stroke care networks. The aim of this project was to test whether it would be possible to implement and achieve a certification of a networkwide quality management system within a large telestroke network, the Stroke Network Using Telemedicine in Northern Bavaria (STENO).

\section{Methods}

\section{Network structure}

STENO is one of the world's largest telemedical stroke care networks, consisting of three tertiary stroke centers in the cities of Erlangen, Nuremberg, and Bayreuth and, currently, 17 local or regional hospitals serving as primary and secondary stroke facilities in the German states of Bavaria and Thuringia, caring for about 5800 acute stroke patients (excluding strokes treated in tertiary centers) per year. Figure 1 shows an organizational diagram of the network structure. The project was started in 2007 based on a preceding pilot study (17) and was fully operational by March 2008. STENO is fully reimbursed by the Bavarian state government and the German health insurance system. Since STENO was established, more than 12000 teleconsultations for patients with assumed acute cerebrovascular disease have been 


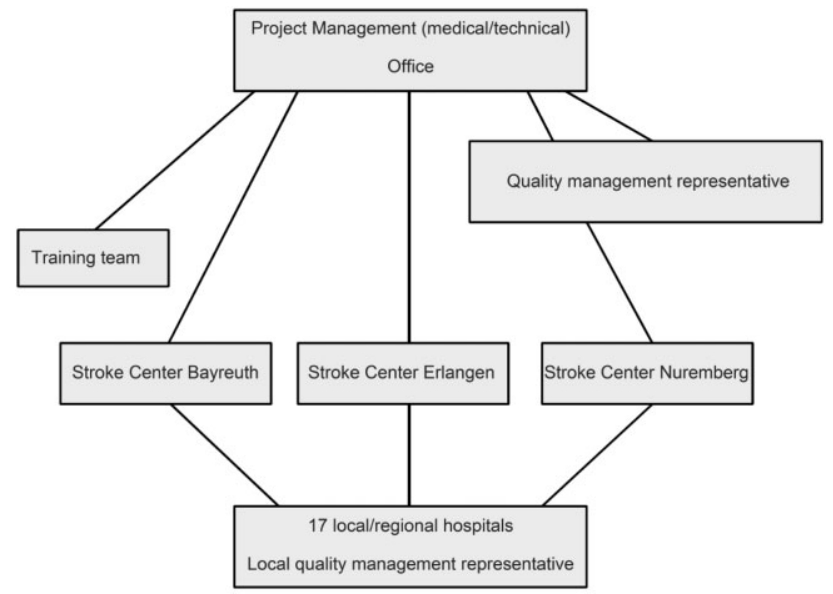

Fig. 1 Organizational diagram of STENO.

Table 1 Requirements for local hospitals participating in the network Minimum requirements

Stroke ward with a minimum of four monitor beds

Stroke team consisting of physician, trained nurses, therapists (physiotherapy, speech therapy, occupational therapy)

CT scanner available 24/7

ECG, echocardiography, Doppler

ultrasound, laboratory available 24/7

Intensive care unit providing mechanical ventilation

Neurology consultation at bedside minimum once weekly

Optional requirements

MRI scanner

Neurologist at site (daily)

Vascular surgery

performed. Thrombolysis has been recommended and performed by the local hospital in more than 600 cases.

Tertiary stroke centers are embedded in large neurology departments of a university hospital (Erlangen) or large teaching hospitals (Nuremberg and Bayreuth). These centers each run a certified stroke unit assigned by the state government according to national guidelines, as well as a designated neurological critical care unit, and have direct access to all additional methods of stroke therapy, including cardiology, neurosurgery, and interventional neuroradiology.

Local hospitals are district hospitals responsible for primary and comprehensive care, primarily in the fields of general medicine and surgery. They vary in size, ranging from about 180 to 450 beds. Accordingly, the number of stroke cases ranges from 200 to 520 per year. In 12 hospitals, the departments of internal medicine are responsible for treatment of stroke, while in three there was a staff neurologist available. Prior to the start of the network, prerequisites for participation were defined (see also Table 1): Each hospital had to have or establish a dedicated stroke ward with monitoring beds and a multidisciplinary stroke care team consisting of physicians, nursing staff, physiotherapists, and occupational and speech and language therapists. Additionally, basic diagnostic equipment like radiology service with a CT scanner, as well as other standard diagnostics such as neurological ultrasound and echocardiography, had to be available in each local hospital on a 24/7 basis. Eligibility of each local hospital to participate in STENO was decided by the state government based on the predefined requirements.

\section{Quality management}

Since the start of the network in 2007, quality management and quality assurance have been part of the core network concept. Quality objectives have been defined, with responsibilities and time targets to be fulfilled. Standard operating procedures (SOPs) were defined for all medical processes and key organizational processes. These SOPs were documented for key indications on table-based charts for all members of the stroke care teams (see example in Fig. 2). All SOPs and other documents and forms were created on the basis of ISO (International Organization for Standardization) 9001:2008 requirements. ISO certification is a voluntary assessment regulated at national, European, and international level (18); health-care accreditation may be voluntary (at national or international level) or mandatory at governmental level (regional or national). Both systems share the principle of assessment of all departments of the hospital by an external visitor or team against published requirements or standards that focus on systems for quality and safety management more than on resources or results. These standards vary within and between countries, especially in accreditation (19); ISO 9001 is a generic standard for quality management systems in any industry, but accreditation standards are specific to health care (20).

On each participating site, an initial training course and repeated lectures were performed for physicians, nurses, therapists, and all other members of the defined stroke team.

Quality control was established by on-site visits and internal audits in each stroke center and local hospital. On-site visits and internal audits were performed in all network institutions by members of the network coordination and training team according to ISO standard 19011:2011. All members of the audit team were trained in methods of quality management. The networkcoordinating team was composed of an experienced vascular neurologist as project leader, a telemedically experienced computer technician as technical leader, and a designated project manager. The training team included a further vascular neurologist, a speech and language therapist, an occupational therapist, a physiotherapist, and a stroke care-certified nurse. Furthermore, a steering committee responsible for the quality management process was established. Additionally, a scheme for routine data collection was predefined. All hospitals participated in the statewide stroke care quality assurance program with quality-of-care indicators and other benchmark data, according to the dataset defined by the German Stroke Registers Study Group (12). Furthermore, special data recording for all teleconsultations and all thrombolysis cases, as well as technical data and service complaints within the network, was implemented. For each teleconsultation, time and duration of consultation, as well as reason and key recommendations, were recorded. For each case of thrombolysis in the network, all timing of process, clinical state prior 


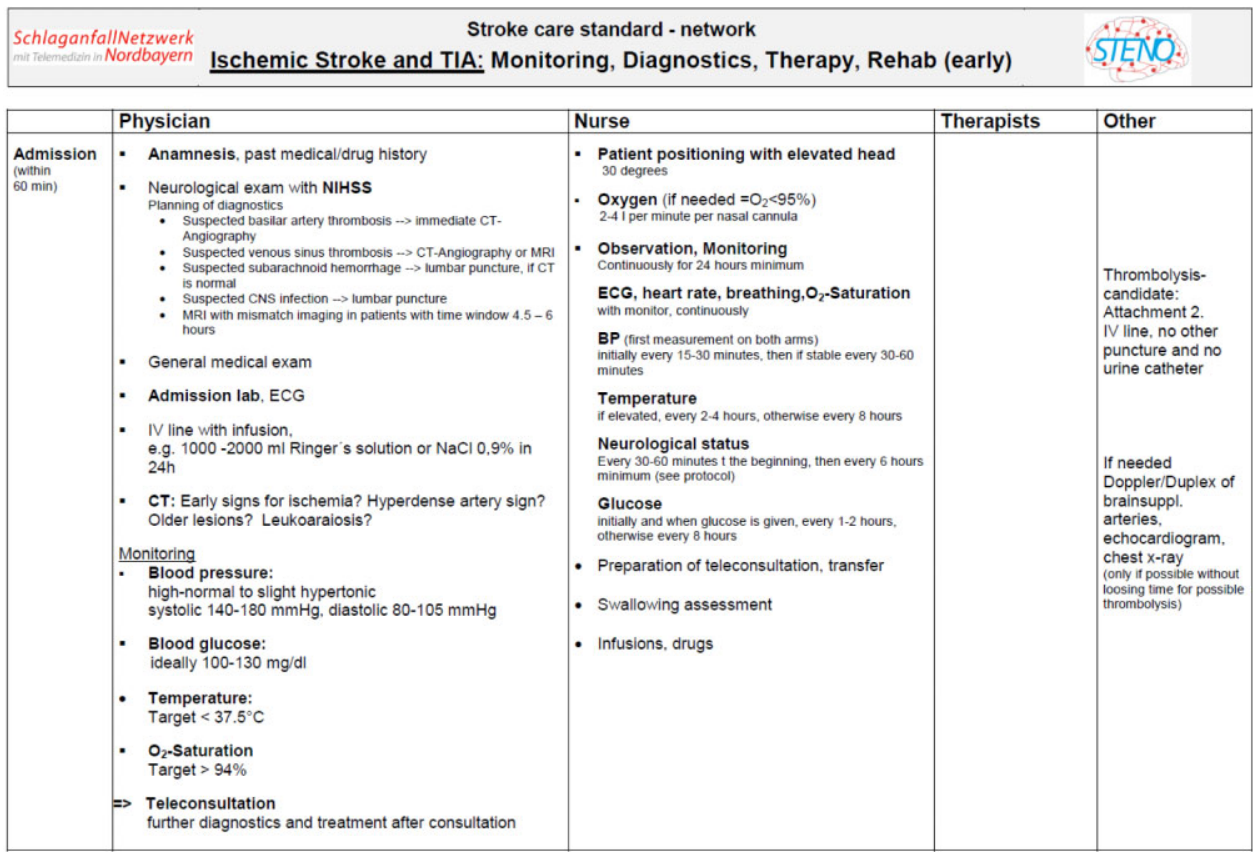

Fig. 2 Example of standard operating procedure: 'Standard therapy: ischemic stroke and TIA' (translated into English).

and after thrombolysis, and outcome at discharge were documented. Once a year, consulting physicians at the centers and physicians in local hospitals as well as patients were interviewed for a period of 4 weeks with a standardized questionnaire concerning customer satisfaction and performance for each individual consultation. In October 2009, a decision was made to accomplish a certification of the quality management system (QMS) by LGA InterCert (TUV Rheinland, Nuremberg, Germany) under the ISO standard 9001:2008. The aim was the implementation and certification of a single quality management system for stroke care in all participating hospitals of the network, including stroke centers and local hospitals. Simultaneously, the GSS developed a revision of its certification standard for stroke units, suitable for units supported by telemedicine.

All SOPs and process descriptions were linked in one quality management manual, together with core descriptions of the network concept, quality objectives, and policies. An auditing and review plan was written, and in 2010, a first management review was published, covering the year 2009. LGA InterCert was chosen as the certification body because it is the only institution covering GSS's stroke unit certification, making a combined certification for ISO 9001 and GSS stroke unit standard possible. The certification process was funded by a grant of the Bavarian State Ministry of Health and Environment.

Internal organization and quality management system Since the start of the network, a general assembly of all participating institutions has been held on a biannual basis and is responsible for all network-relevant decisions. The network management (located at the Erlangen University Hospital) is responsible for all management operations.

The QMS manual was completed in August 2010 and consisted of 37 documents: a core manual with general considerations such as quality policy or organizational framework, all 22 SOPs and other forms, checklists, and worksheets. The medical content of SOPs was based on current standards and guidelines of the GSS, European Stroke Organisation and American Heart Association.

\section{Certification process}

In October 2009, the decision was made to implement one QMS for all institutions in the whole network and to aim for a certification of the network's QMS under one certificate. Accordingly, a contract with LGA InterCert was signed.

In September 2010, all documents, including the completed manual and the management review, were handed over to the certification body. In November 2010, a Phase I audit was conducted at the coordinating and leading stroke center at Erlangen University Hospital, followed by a Phase II audit in January 2011, visiting all three stroke centers as well as five exemplary local hospitals.

In January 2012, another four local hospitals, as well as the project management and all three stroke centers were visited in the first review audit by LGA InterCert. In January 2013, the remaining two local hospitals, one new local hospital that had been added to the network during the certification period, and, again, the project management and all three stroke centers were visited in the second review audit.

Once a year, internal audits were conducted in every participating institution to review the implementation of the QMS and its conformity with the predefined norm.

\section{Results}

\section{Quality performance data}

In 2009, a total 3690 stroke patients were seen treated in all local hospitals combined, of whom 2049 were managed with a teleconsultation, including 1438 with full video examination. In 2011 (2010), a total of 2324 (2047) teleconsultations, including 1695 (1426) video examinations, were made, while a total of 4517 
Table 2 Results of quality indicators in the quality assurance program for years 2009-2011

\begin{tabular}{|c|c|c|c|}
\hline & 2009 & 2010 & 2011 \\
\hline Age, years & $74 \cdot 8$ & 73.9 & $74 \cdot 1$ \\
\hline Independent prior to stroke, \% & $55 \cdot 6$ & $56 \cdot 0$ & $55 \cdot 7$ \\
\hline Admission $<3 \mathrm{~h}, \%$ & $41 \cdot 0$ & $40 \cdot 2$ & 38.6 \\
\hline$C T<60 \min , \%$ & $69 \cdot 7$ & $81 \cdot 1$ & $86 \cdot 3$ \\
\hline Swallowing test, \% & $63 \cdot 4$ & $78 \cdot 1$ & $88 \cdot 2$ \\
\hline Start speech therapy < day 2, \% & $54 \cdot 1$ & $62 \cdot 5$ & $67 \cdot 6$ \\
\hline Physiotherapy, \% & $81 \cdot 6$ & $87 \cdot 8$ & $91 \cdot 3$ \\
\hline Length of stay, days & $7 \cdot 9$ & $7 \cdot 8$ & $7 \cdot 9$ \\
\hline Pneumonia, \% & $3 \cdot 0$ & $3 \cdot 4$ & $4 \cdot 1$ \\
\hline Mortality, \% & $5 \cdot 8$ & $5 \cdot 7$ & $4 \cdot 6$ \\
\hline Rehabilitation, \% & $21 \cdot 1$ & $22 \cdot 2$ & $29 \cdot 1$ \\
\hline Rankin 4-6, \% & $23 \cdot 4$ & $24 \cdot 7$ & $23 \cdot 3$ \\
\hline Institutional care, \% & $9 \cdot 5$ & $10 \cdot 5$ & 8.7 \\
\hline
\end{tabular}

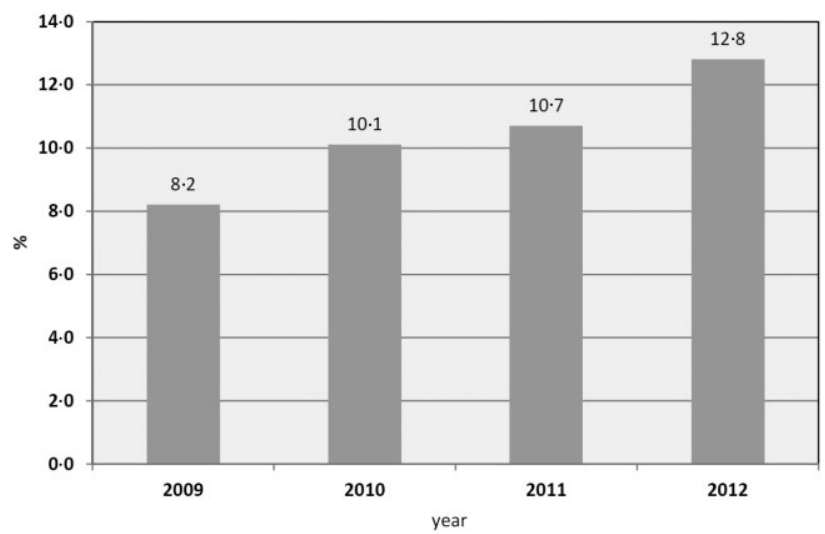

Fig. 3 Rate of thrombolysis in patients with cerebral ischemia from 2009 to 2012.

(4103) patients were seen in all local hospitals combined. Table 2 shows results of several documented quality indicators stratified by year.

Rates of thrombolysis in patients with cerebral ischemia significantly improved from $8 \cdot 2 \%$ in 2009 to $12 \cdot 8 \%(P=0 \cdot 01)$ in 2012, not including patients treated in the three tertiary stroke centers (Fig. 3). Rates of thrombolysis in hospitals prior to their participation in STENO have not been recorded.

\section{Results of external audits}

There was no minor or serious deviation from the norm in the initial certification audit in 2011 or in the two following audits of the certification period in 2012 and 2013. Only a few hints were given by the certification body to ensure further improvement of quality.

In April 2011, the certificate for the network-wide QMS was handed over to the network management.

\section{Results of internal audits}

In 2009, internal audit reports listed a total of 75 deviations at local hospitals and 10 in stroke centers. In 2010, there were 65 deviations in local hospitals and 7 in centers; in 2011, 44 deviations were found locally and 5 in the centers. In Table 3, some examples of reported deviations are given.
Results of quality data, especially the significant improvement of thrombolysis rates, and internal and external audits documented a stepwise improvement indicating a functioning quality management process.

\section{Discussion}

At the beginning of this project, it was not clear if a network consisting of 15 hospitals (with the potential of expansion), different in size and organizational models of stroke care, would be able to implement and follow the the ISO 9001 requirements and to maintain this level of quality throughout the whole network in order to hold the certification for a longer period of time. In fact, certification was awarded in March 2011 and maintained over a total certification cycle of three-years without major deviation concerning nonconformity with the norm in three external thirdparty audits.

Creating a QMS based on ISO 9001 requirements was the cornerstone of sustainable quality assurance within STENO. A common high level of stroke care was defined by quality objectives and SOPs describing processes that led toward predefined goals. The QMS guaranteed utilization of SOPs and other process regulations within the network by internal and external audits in every participating institution. Continuous documentation and monitoring of data quality ensured permanent accomplishment of key quality objectives.

ISO 9001 is a common quality standard that is used worldwide in almost every kind of industry or service organization (21). Thus, ISO 9001 requirements focus on more formal parts of quality management but less on specific quality levels of stroke care particularly or medicine in general. Therefore, the combination of network certification with certification of individual stroke units by the standards of the GSS in each participating hospital provided an excellent basis for combining formal quality requirements with medical and clinical requirements in all participating institutions. While SOPs in STENO were based on national and international guidelines for stroke care, stroke unit certification audits revealed many details, with improvement potential in all kind of processes.

STENO is based on the use of telemedicine - especially audiovisual consultation - in single acute stroke cases as one main column of the comprehensive stroke care system. However, the STENO QMS could serve as a model for cooperative quality assurance of stroke care in cooperating hospitals because the QMS would also be effective in a network without using telemedicine. Only a minor part of STENO regulations and procedures deal with telemedicine itself rather than medical care in general and stroke care in particular. Nevertheless, teleconsultations may provide quality assurance in themselves, as they actually constitute a review of a single case by a stroke expert. Within the consultation, the stroke expert defines which standard is used in the particular case and if variations of the standard are judged to be necessary for the individual patient based on the expert's personal knowledge and experience. Additionally, securing the same method and level of assessment and advice from various experts 
Table 3 Deviations/key notes for improvement in internal audits from 2009 to 2011

\begin{tabular}{|c|c|c|c|c|}
\hline & \multicolumn{2}{|l|}{ Local hospitals } & \multicolumn{2}{|c|}{ Stroke centers/network coordination } \\
\hline & Minor/serious & Examples & Minor/serious & Examples \\
\hline 2009 & $\begin{array}{l}75 / 0 \\
\text { (all } 11 \text { hospitals) }\end{array}$ & $\begin{array}{l}\text { - Stroke nursing staff needs training } \\
\text { - Documentation of thrombolysis should be } \\
\text { more standardized } \\
\text { - Availability of documents on stroke ward } \\
\text { must become more consistent }\end{array}$ & $\begin{array}{l}10 / 0 \\
\text { (all } 3 \text { centers) }\end{array}$ & $\begin{array}{l}\text { - Introductory training for all stroke fellows } \\
\text { in telemedicine service must be } \\
\text { completed } \\
\text { - A different phone is needed to secure } \\
\text { availability for teleconsultation }\end{array}$ \\
\hline 2010 & $\begin{array}{l}65 / 0 \\
\text { (7 hospitals) }\end{array}$ & $\begin{array}{l}\text { - Stroke patients should be concentrated in } \\
\text { one ward after monitoring } \\
\text { - Neurologist should be integrated in ward } \\
\text { rounds }\end{array}$ & $\begin{array}{l}7 / 0 \\
\text { (all } 3 \text { centers) }\end{array}$ & $\begin{array}{l}\text { Regular meetings and lectures for } \\
\text { teleconsulting experts need to be } \\
\text { established }\end{array}$ \\
\hline 2011 & $\begin{array}{l}44 / 0 \\
\text { ( } 5 \text { hospitals) }\end{array}$ & $\begin{array}{l}\text { - A higher frequency of team meetings is } \\
\text { needed } \\
\text { - Swallowing test and nutrition type need } \\
\text { documentation }\end{array}$ & $\begin{array}{l}5 / 0 \\
\text { (all } 3 \text { centers) }\end{array}$ & $\begin{array}{l}\text { - Availability of STENO-SOPs at } \\
\text { teleconsultation unit must be improved }\end{array}$ \\
\hline
\end{tabular}

from three different stroke centers represents another challenging task for quality management.

Similarly to the certification system used in STENO combining ISO 9001 and medical requirements, the German Cancer Society successfully promotes nationwide certifications of breast cancer centers (22). By contrast, in stroke care, it is predominantly national review standards or certifications of care systems/ facilities such as the National Stroke Audit (Great Britain) (23) or the Certification of Primary or Comprehensive Stroke Centers by the JCAHO (USA) that are established. JCAHO certification of stroke centers was shown to reduce mortality in certified institutions (24) but did not affect the rate of intravenous thrombolysis (25). Due to lack of expert knowledge and deficits in specialized care, there was almost no systemic thrombolysis therapy in most regional hospitals before STENO was implemented. However, after implementation of essential hospital infrastructure, procedures, and standardized stroke care, thrombolysis is now common and performed on a regular basis in all participating hospitals, significantly reducing the burden of the disease for those affected and for whole communities.

There are a few methodological issues that merit discussion. First, due to the size of the network, organizational issues, and financial restrictions, it was not possible to perform external third-party audits on a yearly basis in each participating hospital. Based on the regulations of ISO 9001 and ISO 17021, it was decided to visit each center and the network management every year and each regional hospital once in the certification period of three-years. Second, our comprehensive approach only covers the acute and subacute phases of stroke from admission to hospital until discharge to rehabilitation or premorbid living environment. However, we believe there is great potential for an expansion of our comprehensive approach by including rehabilitation - ambulatory or inpatient care - or even community care in our QMS. Finally, in order to demonstrate the significance of the implemented changes to stroke care in a whole region, it would have been interesting to analyze comparative outcome and performance data from pre- and postimplementation periods. However, the aim of this project was to test whether it was pos- sible to implement and achieve a certification of a network-wide QMS. Further analysis of performance and outcome data is on its way and will be dealt with in a future manuscript.

In conclusion, quality management within a large supraregional stroke network is possible. Implementing the worldrenowned ISO 9001 certification requirements could enforce a more systematic and continuous approach of quality management and may facilitate development of an international standard for certification of stroke care facilities within networks using teleconsultation techniques. Furthermore, the recently published European norm EN 15224, which has been created especially for the medical sector may be even more beneficial due to its medicine-specific content, as opposed to having an industrybased origin. Furthermore, to improve the availability and the quality of affordable stroke care, the European Stroke Organisation (ESO) has appointed an ESO Stroke Unit Certification Committee to define the requirements and criteria for official certification as ESO Stroke Units and ESO Stroke Centers based on scientific evidence from randomized controlled trials, clinical practice guidelines, and expert consensus. Their recommendations have been published (2013) and should stimulate hospitals in Europe to apply for ESO certification (26).

Telemedical stroke care networks seem to benefit from quality management and certification, as these require continuous effort for quality maintenance and strengthen internal and transinstitutional, as well as multidisciplinary, cooperation. However, outcome studies comparing conventional stroke care and telestroke care are inevitable.

\section{References}

1 Medcalf RL, Davis SM. Plasminogen activation and thrombolysis for ischemic stroke. Int J Stroke 2012; 7:419-25.

2 Shafqat S, Kvedar CJ, Guanci MM, Chang EC, Schwamm LH. Role for telemedicine in acute stroke: feasibility and reliability of remote administration of the NIH Stroke Scale. Stroke 1999; 30:2141-214.

3 Handschu R, Littmann R, Reulbach U et al. Telemedicine in emergency evaluation of acute stroke: interrater agreement in remote video examination with a novel multimedia system. Stroke 2003; 34:2842-6. 
4 Wang S, Lee SB, Pardue C et al. Remote evaluation of acute ischemic stroke: reliability of National Institutes of Health Stroke Scale via telestroke. Stroke 2003; 34:e188-91.

5 Wiborg A, Widder B. Teleneurology to improve stroke care in rural areas: the Telemedicine in Stroke in Swabia (TESS) Project. Stroke 2003; 34:2951-6.

6 La Monte M, Bahout M, Hu P et al. Telemedicine for acute stroke. Triumphs and pitfalls. Stroke 2003; 34:725-8.

7 Audebert HJ, Schenkel J, Heuschmann PU, Bogdahn U, Haberl RL. Effects of the implementation of a telemedical stroke network: the Telemedic Pilot Project for Integrative Stroke Care (TEMPiS) in Bavaria, Germany. Lancet Neurol 2006; 5:742-8.

8 Audebert HJ, Schultes K, Tietz V et al. Long-term effects of specialized stroke care with telemedicine support in community hospitals on behalf of the telemedical project for integrative stroke care (TEMPIS). Stroke 2009; 40:902-8.

9 Donabedian A. Evaluating the quality of medical care. 1966. Milbank Quart 2005; 83:691-729.

10 Donabedian A. The quality of medical care. Science 1987; 200:856-64.

11 Nabavi DG, Ringelstein EB, Faiss J, Kessler C, Röther J, Busse O. Regional and national stroke units in Germany: amended certification criteria. Nervenarzt 2012; 83:1039-52.

12 Heuschmann PU, Biegler MK, Busse O et al. Development and implementation of evidence-based indicators for measuring quality of acute stroke care: the Quality Indicator Board of the German Stroke Registers Study Group (ADSR). Stroke 2006; 37:2573-8.

13 Schwamm LH. When in Rome, do like the Romans: certifying stroke centers with the rod of Aesculapius or the medical caduceus of Hermes? J Am Heart Assoc 2013; 2:e000120.

14 Alberts MJ, Latchaw RE, Jagoda A et al. Revised and updated recommendations for the establishment of primary stroke centers: a summary statement from the Brain Attack Coalition. Stroke 2011; 42:2651-65.

15 Leifer D, Bravata DM, Connors JJ III et al. Metrics for measuring quality of care in comprehensive stroke centers: detailed follow-up to Brain Attack Coalition comprehensive stroke center recommendations: a statement for healthcare professionals from the American Heart Association/American Stroke Association. Stroke 2011; 42:84977.
16 Lichtman JH, Allen NB, Wang Y, Watanabe E, Jones SB, Goldstein LB. Stroke patient outcomes in US hospitals before the start of the Joint Commission Primary Stroke Center certification program. Stroke 2009; 40:3574-9.

17 Handschu R, Scibor M, Willaczek B et al. Telemedicine in acute stroke: remote videoexamination compared to simple telephone consultation. J Neurol 2008; 255:1792-7.

18 European Union. Regulation (EC) 765/2008 of the European Parliament and of the Council of 9 July 2008 setting out the requirements for accreditation and market surveillance relating to the marketing of products. 2008. Available at: http://eurlex.europa.eu/LexUriServ/ LexUriServ.do?uri=OJ:L:2008:218:0030:0047:en:PDF.

19 Shaw CD, Bruneau C, Kutryba B et al. Towards hospital standardization in Europe. Int J Qual Health Care 2010; 22:244-9.

20 Shaw CD, Groene O, Botje D etal. The effect of certification and accreditation on quality management in 4 clinical services in 73 European hospitals. Int J Qual Health Care 2014; 26(Suppl. 1):100-7.

21 International Organization for Standardization. ISO 9001:2008 (en) Quality management systems - requirements. 2008. Available at: https://www.iso.org/obp/ui/\#iso:std:iso:9001:ed-4:v1:en (accessed 20 August 2013).

22 Wallwiener M, Brucker SY, Wallwiener D; Steering Committee. Multidisciplinary breast centres in Germany: a review and update of quality assurance through benchmarking and certification. Arch Gynecol Obstet 2012; 285:1671-83.

23 Rudd AG, Hoffman A, Irwin P, Lowe D, Pearson MG. Stroke unit care and outcome: results from the 2001 National Sentinel Audit of Stroke (England, Wales, and Northern Ireland). Stroke 2005; 36:103-6.

24 Lichtman JH, Jones SB, Wang Y, Watanabe E, Leifheit-Limson E, Goldstein LB. Outcomes after ischemic stroke for hospitals with and without joint commission-certified primary stroke centers. Neurology 2011; 76:1976-82.

25 Mullen MT, Kasner SE, Kallan MJ, Kleindorfer DO, Albright KC, Carr BG. Joint commission primary stroke centers utilize more rt-PA in the nationwide inpatient sample. J Am Heart Assoc 2013; 2:e000071.

26 Ringelstein EB, Chamorro A, Kaste M et al. European Stroke Organisation recommendations to establish a stroke unit and stroke center. Stroke 2013; 44:828-40. 